\title{
Relationship Between Circulating E-Selectin, DD Genotype of Angiotensin-Converting-Enzyme, and Cardiovascular Damage in Central Obese Subjects
}

\author{
Giuseppe Licata, Tiziana Di Chiara, Anna Licata, Giovanni Triolo, Christiano Argano, Antonio Pinto, \\ Gaspare Parrinello, Salvatore Corrao, Giovanni Duro, and Rosario Scaglione
}

\begin{abstract}
Fifty-six young central obese patients were investigated to evaluate relationships between soluble E-selectin (sE-S), angiotensin-converting enzyme (ACE) gene polymorphism, left ventricular function and structure, and carotid morphology by determination of sE-S and ACE genotypes. Our results indicated that central obese subjects with concomitant higher levels of sE-S and ACE DD genotype may be characterized by early cardiovascular alterations and then considered a particular subset of subjects at higher risk of cardiovascular disease.
\end{abstract}

(c) 2003 Elsevier Inc. All rights reserved.

$\mathbf{C}$ ENTRAL OBESITY has to be considered today an emerging risk factor for atherosclerotic cardiovascular disease. ${ }^{1-3}$ In fact, an atherogenic metabolic profile, ${ }^{4}$ a prothrombotic hypofibrinolitic pattern, ${ }^{5}$ an altered sodium-regulating hormone control, ${ }^{6}$ and a silent left ventricular dysfunction ${ }^{7,8}$ may be detectable early in young central obese subjects.

On the other hand, it is known that structural or functional changes in vascular endothelium are involved in the pathogenesis of atherosclerotic vascular diseases. In fact, it has to be emphasized that endothelium may react to several stimuli with dysfunctional responses. ${ }^{9}$

An impaired endothelium-dependent vasodilation has been reported not only in clinically overt atherosclerosis, ${ }^{10}$ but also in some subsets of patients at higher cardiovascular risk such as hypertensives, hyperdyslipidemics, and diabetics. ${ }^{10-12}$ This abnormality is consistent with a rapid adhesion and aggregation of platelet at the sites of the injury, and in the activation of the coagulation cascade. ${ }^{9,13}$ It has recently been reported that endothelial function may be evaluated looking for some endothelial molecules that are expressed and amplified when an endothelial injury occurs. Accordingly, an important role has been attributed to endothelium-bound E-selectin. In fact, this endothelium-specific molecule is able to mediate the initial phase (rolling) of adhesion of leukocytes to endothelium.9,14 In addition, soluble E-selectin (sE-S) has been recently proposed as a new and early marker of endothelial injury/damage. ${ }^{15-17}$

Moreover, despite the role of angiotensin-converting enzyme (ACE), DD genotype as a cardiac risk factor remains controversial. ${ }^{18-20}$ The $\mathrm{D}$ allele has been consistently associated with higher ACE activity or angiotensin II levels across distinct patient populations, including hypertensive and central obese subjects. ${ }^{20,21}$ The changes in ACE activity associated with the $\mathrm{D}$ allele could affect endothelial function by 2 potential mechanisms. First, an increase in plasma ACE may promote angiotensin I to angiotensin II conversion and therefore amplification of the generation of superoxide anions that degrade nitric oxide (NO). Second, high ACE levels may increase bradikynin degradation and therefore reduce bradykynin-induced NO effects. ${ }^{22}$ Although these data suggest a pathophysiological connection between the D allele and endothelial dysfunction, at present, few data are available on the relation between central obesity, endothelial function, ACE genotypes, and atherosclerosis. The current study was designed to analyze the pattern of sE-S and ACE genotypes in young central obese subjects without major risk factors for cardiovascular disease and their relationships with early markers of cardiovascular damage. The main goal of the study was to evaluate whether higher circulating E-selectin values and homozygosity for the D allele of ACE could contribute to the early recognition subset of central obese subjects with a higher susceptibility to atherosclerotic cardiovascular disease.

\section{MATERIALS AND METHODS}

\section{Subjects}

A total of 56 obese subjects younger than 40 years of age with central fat distribution were included in the study. Obese subjects were consecutively recruited in the obesity center of the Internal Medicine Department at the University of Palermo (Italy). The subjects were classified as obese according to sex-specific 85 th percentile of body mass index (BMI) values, as reported at the Italian Consensus Conference on Obesity. ${ }^{23}$ Accordingly, males with a BMI greater than 30.5 $\mathrm{kg} / \mathrm{m}^{2}$ and females with a BMI greater than $27.3 \mathrm{~kg} / \mathrm{m}^{2}$ were considered obese. Body fat distribution was evaluated by measuring waist-to-hip ratio (WHR) in the standing position, as previously reported. ${ }^{24,25} \mathrm{Cen}-$ tral obesity was defined according to the sex-specific 85th percentile of WHR values. Males with WHR values $\geq 0.92$ and females with WHR values $\geq 0.81$ were considered to have central obesity. ${ }^{4,23-25}$

Accordingly, accurate family and clinical history and laboratory investigations were obtained. Exclusion criteria were smoking habits, hypertension (according to International Society of Hypertension [ISH] guidelines, arterial pressure was recorded with an appropriate large cuff in obese subjects), cardiovascular diseases (defined as myocardial infarction, chest pain, heart block, valvular disease, and heart failure), renal disease, insulin-dependent or -independent diabetes mellitus, familial hyperdyslipidemia, electrolyte imbalances, alcoholism, or psychiatric problems. The study was approved by the Ethics Committee of University of Palermo School of Medicine and each patient gave

From the Department of Internal Medicine, University of Palermo; Unit of Clinical Methodology, Epimediological and Statistics, National Relevance Hospital Trust, Civico e Benfratelli, Palermo; and the National Research Center, Palermo, Italy.

Submitted July 6, 2002; accepted March 31, 2003.

Supported by a project grant from Ministero dell'Università e della Ricerca Scientifica (Italy).

Address reprint requests to Rosario Scaglione, MD, Associate Professor of Medical Therapy, Via Lombardia n. 9, 90144, Palermo, Italy. (c) 2003 Elsevier Inc. All rights reserved.

0026-0495/03/5208-0007\$30.00/0

doi:10.1016/S0026-0495(03)00150-1 
informed consent after receiving a detailed description of the study procedure. Preliminary investigations included measurements of blood and urinary electrolytes, creatinine clearance, and blood sugar both basal and after an oral glucose tolerance test. In addition, basal immunoreactive insulin levels (IRIb) and 2-hour post-glucose tolerance test (2h-IRI) values were recorded.

\section{Methods}

Measurements ofsE-S. sE-S was measured in all sera by sandwich enzyme immunoassay purchased from Bender Medsystem (Vienna, Austria) following the manufacturer's instructions. Sensitivity, and interassay and intra-assay variability were validated in our laboratory and were comparable to those obtained by the manufacturer. ${ }^{26}$ Results were expressed as nanograms per milliliter.

Levels of sE-S were determined in all subjects and grouped according to level: less than $80 \mathrm{ng} / \mathrm{mL}$ (group A) and $\geq 80 \mathrm{ng} / \mathrm{mL}$ (group B). Group A patients with a normal sE-S value consisted of 42 central obese subjects ( 29 men and 22 women), with a mean sE-S value of $54 \pm 13$ and a mean age of $33 \pm 5$ years. Group B patients with higher sE-S values consisted of 14 central obese subjects $(6$ men and 8 women) with a mean sE-S value of $99 \pm 15$ and a mean age of $35 \pm$ 3 years.

The cut-off value of sE-S was related to the mean value $\pm 2 \mathrm{SD}$ of young healthy control sample (without a family history of premature cardiovascular disease) from our laboratory. ${ }^{26}$ Our mean values reported in normal subjects are in agreement with those reported by others. 27,28

Determination of ACE genotypes. The D and I alleles were identified on the basis of polymerase chain reaction (PCR) amplification of the respective fragments from intron 16 of the ACE gene and size fractionation and visualization by electrophoresis gel. For preparing PCR templates from human whole blood, we used the Split Second DNA Preparation Kit (Boehringer Mannheim, Germany).

We used an optimized primer pair to amplify the D and I alleles, resulting in 319-bp and 597-bp amplicons, respectively, hace3s and hace3as. The amplification products of the $\mathrm{D}$ and I alleles were identified by $300-\mathrm{nm}$ ultraviolet transillumination as distinct bands; in heterozygous samples, a third band, assumed to represent a heteroduplex DNA product, was commonly seen.

Because the $\mathrm{D}$ allele in heterozygous is amplified preferentially, each sample found to have the DD genotype was subjected to a second independent PCR amplification with a primer pair that recognizes an insertion-specific sequence, hace $5 \mathrm{a}$ and hace $5 \mathrm{c}$, with identical PCR conditions except for an annealing temperature of $67^{\circ} \mathrm{C}$. The reaction yields a 335-bp amplicon only in the presence of an I allele, and no product in samples homozygous for DD. This procedure correctly identified the $4 \%$ to $5 \%$ of samples with the DI genotype that are misclassified as DD with the insertion-spanning primers.

Casual and 24-hour blood pressure. Casual systolic and diastolic blood pressure (SBPc and $\mathrm{DBPc}$ ) were measured in triplicate by mercury sphygmomanometer with subjects in the supine position. DBPc refers to Korotkoff $\mathrm{V}$ phase. Casual mean blood pressure (MBPc) was calculated from the sum of DBPc plus one third of the arterial pulse pressure. Arterial pressure was measured with an appropriately wide cuff in all subjects. Heart rate was evaluated by electrocardiographic tracing. ${ }^{4,29,30}$

Ambulatory blood pressure was recorded by the portable fully automatic Takeda (Japan) TM2420 system connected through the serial interface (RS 232) to an IBM (Italy) personal system 2 computer, which in our laboratory showed a correlation of $r=0.96$ for both SBPc and DBPc. ${ }^{23,24,29}$ An appropriately large cuff was available to allow precise 24-hour blood pressure readings in obese subjects. This approach was well tolerated by all patients. The 24 -hour mean SBP, DBP, and MBP were calculated.
Echocardiographic measurements. Left ventricular mass (LVM), LVM indexed for the body height (LVM/h), and cardiac output (CO) were calculated by echocardiographic findings, as previously reported. ${ }^{25,31}$

Two-dimensional and M-mode echocardiography examination was performed by an Esaote Biomedica (Italy) computer-aided ultrasound system equipped with $2.5-$ and $3.5-\mathrm{MHz}$ faced array transducers and a standard VHS video system.

Left ventricular ejection fraction (LVEF) by left ventricular end diastolic and end systolic volumes was measured from the apical 4-chamber view, using the ellipsoid single-plane algorithm. Mean EF was automatically calculated by the echocardiography processing system. In our laboratory the EF calculated on 5 consecutive beats permitted optimal reproducibility and accuracy. ${ }^{31}$

Total peripheral resistance (TPR) was calculated by the formula: $\mathrm{MBP} \times 80 \times 1,332 / \mathrm{CO}$ and expressed as dynes $\times \mathrm{s} \times \mathrm{cm}^{-5}$.

Carotid measurements. In all the subjects, an ultrasound highresolution B-mode imaging examination of the common carotid arteries with scanning on the longitudinal axis until the bifurcation and on the transversal axis was performed using an instrument generating a wideband ultrasonic pulse with a middle frequency of $7.5 \mathrm{MHz}$ (Toshiba SSA-270 ${ }^{\circ}$, Tokyo, Japan).

Intima media wall thickness (IMWT) and lumen diameter (LD) of right and left carotid arteries were calculated according to a method well used and standardized in our laboratory in obese subjects as previously reported. ${ }^{32}$

\section{Statistical Analysis}

Values are expressed as the mean \pm SD. Differences in measurements between the 2 obese groups were analyzed by unpaired $t$ test after unequal variances analysis. Differences in the prevalence of DD ACE genotype and insulin-resistant obese subjects in both groups were analyzed by chi-square test. Linear and multiple regression analyses were used to calculate correlation coefficients between sE-S, BMI, WHR, BP, and measurements of left ventricular structure and function and of carotid morphology. $P$ values $<.05$ were considered statistically significant.

\section{RESULTS}

Central obese subjects were subdivided into 2 groups with normal $(<80 \mathrm{ng} / \mathrm{mL})$ and higher $(\geq 80 \mathrm{ng} / \mathrm{mL})$ sE-S values. The 2 groups were comparable for gender, age, and height (Table 1$)$. In contrast, BMI $(P<.01)$, WHR $(P<.01)$, IRI $(P<.001)$, and 2 h-IRI $(P<.001)$ levels were significantly higher in central obese subjects with higher sE-S values(group $\mathrm{B})$. In addition, the percentage of subjects with insulin resistance $(P<.001)$ and with DD ACE genotype $(P<.01)$ was significantly higher in group B than in group A (Table 1).

\section{Hemodynamic, Cardiac, and Carotid Measurements}

Casual and ambulatory mean blood pressure $(P<.05)$, TPR $(P<.05) \mathrm{LVM} / \mathrm{h}(P<.001)$, and IMWT of both carotid arteries $(P<.05)$ were significantly higher, whereas LVEF $(P<.01)$ were significantly lower in central obese subjects with higher sE-S values than in subjects with normal sE-S values (Table 2).

\section{Correlation}

In all central obese subjects $\mathrm{sE}-\mathrm{S}$ values were directly correlated with WHR $(r=0.59 ; P<.001), \mathrm{LVM} / \mathrm{h}(r=0.51 ; P<$ $.001)$, and 2h-IRI $(r=0.55 ; P<.001)$, and inversely correlated 
Table 1. Clinical Characteristic, Basal and After Oral Glucose Tolerance Test Insulin Levels, and Prevalence in ACE Genotypes Between the Two Groups of Central Obese Subjects

\begin{tabular}{lccc}
\hline & $\begin{array}{c}\text { Group A, } \\
\text { E-Selectin } \\
<80 \mathrm{ng} / \mathrm{mL}\end{array}$ & $\begin{array}{c}\text { Group B, } \\
\text { E-Selectin } \\
\geq 80 \mathrm{ng} / \mathrm{mL}\end{array}$ & $P<$ \\
\hline No. of cases & 42 & 14 & \\
M/F & $20 / 22$ & $6 / 8$ & \\
Age (yr) & $33 \pm 5$ & $35 \pm 3$ & $\mathrm{NS}$ \\
Height (cm) & $161 \pm 9$ & $164 \pm 8$ & $\mathrm{NS}$ \\
BMI (kg/m $\left.{ }^{2}\right)$ & $34 \pm 3$ & $38 \pm 5$ & .01 \\
WHR (\%) & $0.90 \pm 0.07$ & $1 \pm 0.05$ & .01 \\
sE-S (ng/mL) & $54 \pm 13$ & $99 . \pm 15$ & .001 \\
IRIb (pmol/L) & $13 \pm 8$ & $22 \pm 9$ & .001 \\
2h-IRI (pmol/L) & $600 \pm 238$ & $994 \pm 367$ & .001 \\
IR (\%) & $28(10 / 42)$ & $79(11 / 14)$ & $.001^{*}$ \\
DD genotype (\%) & $36(15 / 42)$ & $71(10 / 14)$ & $.01 *$ \\
DI genotype (\%) & $28(12 / 42)$ & $22(3 / 14)$ & $\mathrm{NS}$ \\
II genotype (\%) & $36(15 / 42)$ & $7(1 / 14)$ & $.01^{*}$ \\
\hline
\end{tabular}

Abbreviations: ACE, angiotensin converting enzyme; $M / F$, males/ females; BMI, body mass index; WHR, waist-hip ratio; $\mathrm{sE}-\mathrm{S}$, soluble E-selectin; IRIb, basal insulin; 2h-IRI, 2-hour insulin after oral glucose tolerance test; IR, insulin-resistance; NS, not significant.

${ }^{*}$ Chi-square test.

with LVEF ( $r=-0.69 ; P<.001)$ (Figs 1 and 2). Multiple regression analysis indicated that sE-S levels were independently related to LVM/h and LVEF also when age, BMI, and 24-hour MBP were included in the analysis, but only in subjects with the ACE DD genotype (Table 3 ).

\section{DISCUSSION}

The detection of early alterations in left ventricular structure and function occurs commonly in central obese subjects, as reported by us in previous studies. $4,7,8,29$ The results of present study indicate that there is a clear association between sE-S, insulin levels, blood pressure, LVM and function, and carotid wall thickness in centrally obese subjects. In particular, higher

Table 2. Hemodynamic, Cardiac, and Carotid Measurements in the Two Groups of Central Obese Subjects

\begin{tabular}{lccc}
\hline & $\begin{array}{c}\text { Group A, } \\
\text { E-Selectin } \\
<80 \mathrm{ng} / \mathrm{mL}\end{array}$ & $\begin{array}{c}\text { Group B, } \\
\text { E-Selectin } \\
\geq 80 \mathrm{ng} / \mathrm{mL}\end{array}$ & $P<$ \\
\hline No. of cases & 42 & 14 & \\
MBPc (mm Hg) & $90 \pm 8$ & $98 \pm 7$ & .05 \\
MBP/24 h (mm Hg) & $90 \pm 7$ & $97 \pm 6$ & .05 \\
HR $\left(\mathrm{b} / \mathrm{m}^{2}\right)$ & $76 \pm 10$ & $75 \pm 8$ & $\mathrm{NS}$ \\
TPR $\left(\right.$ dynes s $\left.\times \mathrm{cm}^{-5}\right)$ & $1,870 \pm 470$ & $2,190 \pm 570$ & .05 \\
LVEF $(\%)$ & $61 \pm 2$ & $56 \pm 3$ & .01 \\
LVM/h $(\mathrm{g} / \mathrm{m})$ & $87 \pm 15$ & $105 \pm 15$ & .001 \\
IMWT/sn (mm) & $0.60 \pm 0.16$ & $0.69 \pm 0.2$ & .05 \\
IMWT/dx (mm) & $0.64 \pm 0.12$ & $0.73 \pm 0.2$ & .05 \\
LD dx $(\mathrm{mm})$ & $6.2 \pm 0.8$ & $6.3 \pm 0.9$ & $\mathrm{NS}$ \\
LD sn $(\mathrm{mm})$ & $6.2 \pm 0.7$ & $6.4 \pm 0.6$ & $\mathrm{NS}$ \\
\hline
\end{tabular}

Abbreviations: $\mathrm{MBPc}$, casual mean blood pressure; $\mathrm{MBP} / 24 \mathrm{~h}, 24-$ hour MBP; HR, heart rate; TPR, total peripheral resistance; LVEF, left ventricular ejection fraction; LVM/h, left ventricular mass/height; IMWT, intima-media wall thickness of left (sn) and right $(\mathrm{dx})$ carotid; LD, lumen diameter.
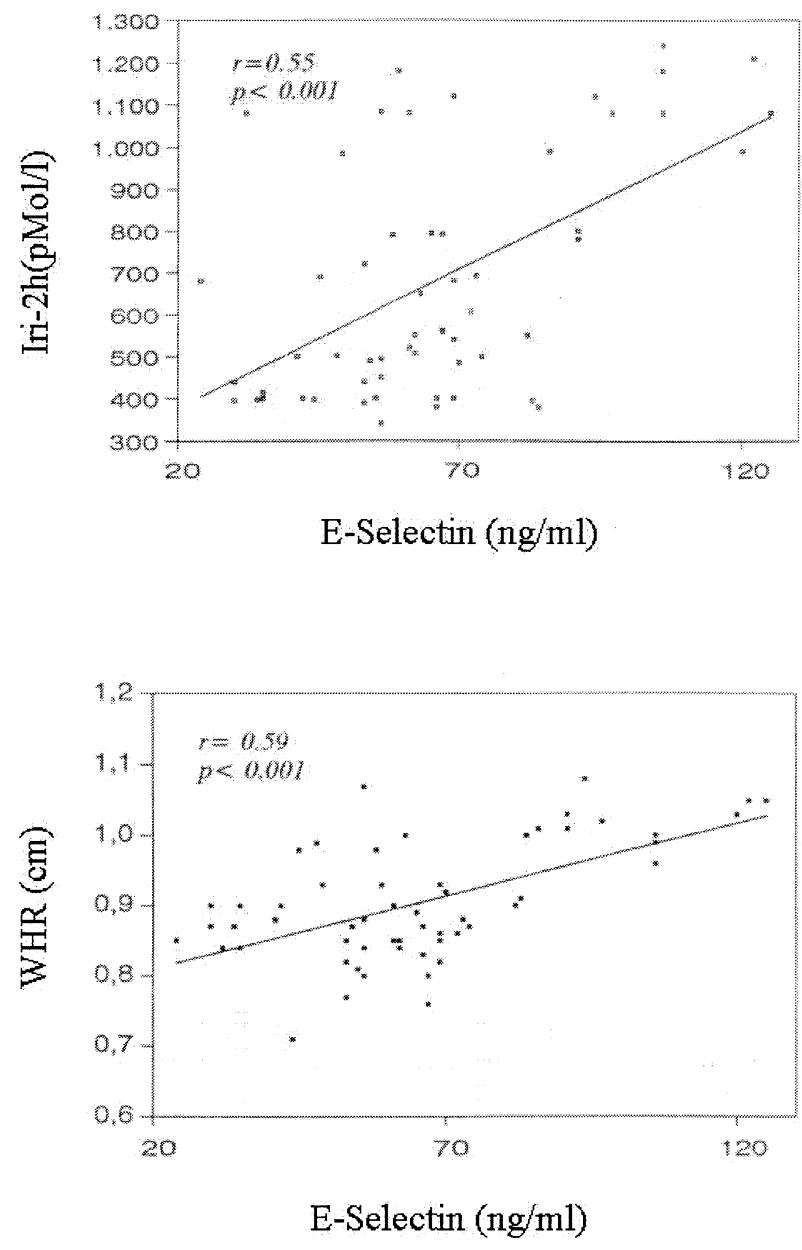

Fig 1. Relationship between sE-S levels, 2 hours after oral glucose tolerance test insulin levels (IRI-2h), and WHR in all subjects studied.

values of BMI, WHR, 2h-IRI, MBPc, 24-hour MBP, LVM/h, IMWT of both carotid arteries, and TPR, and lower values of LVEF, were found in group B (higher sE-S) than in group A (normal sE-S). Several data demonstrated that endotheliumbound E-selectin and other adhesion molecules have been detected more commonly in human atherosclerotic lesions than in healthy arterial tissue. ${ }^{14,33,34}$ This fact might be explained by the inflammatory, immune, and hemostatic actions attributed to endothelium-bound E-selectin under condition of shearstress.9,14 This further supports the idea that atherosclerosis may, in part, be an inflammatory disease. Experimental data suggest that knock-out mice deficient in E-selectin, P-selectin, or intracellular adhesion molecule-1 (ICAM-1) develop fewer arterial lesions than normal mice. ${ }^{33,34}$ Although increased cell surface expression of adhesion molecules is difficult to quantify in vivo, soluble forms can now been measured in serum. ${ }^{35}$ In this context there are limited and contrasting clinical data about the predictive role of circulating adhesion molecules and also of sE-S on cardiovascular atherosclerotic disease. In fact, recent results from a methanalysis of Malick et $\mathrm{al}^{36}$ do not demonstrate a significant role of selectin molecules on cardiovascular risk. Indeed, results from Atherosclerosis Risk in 

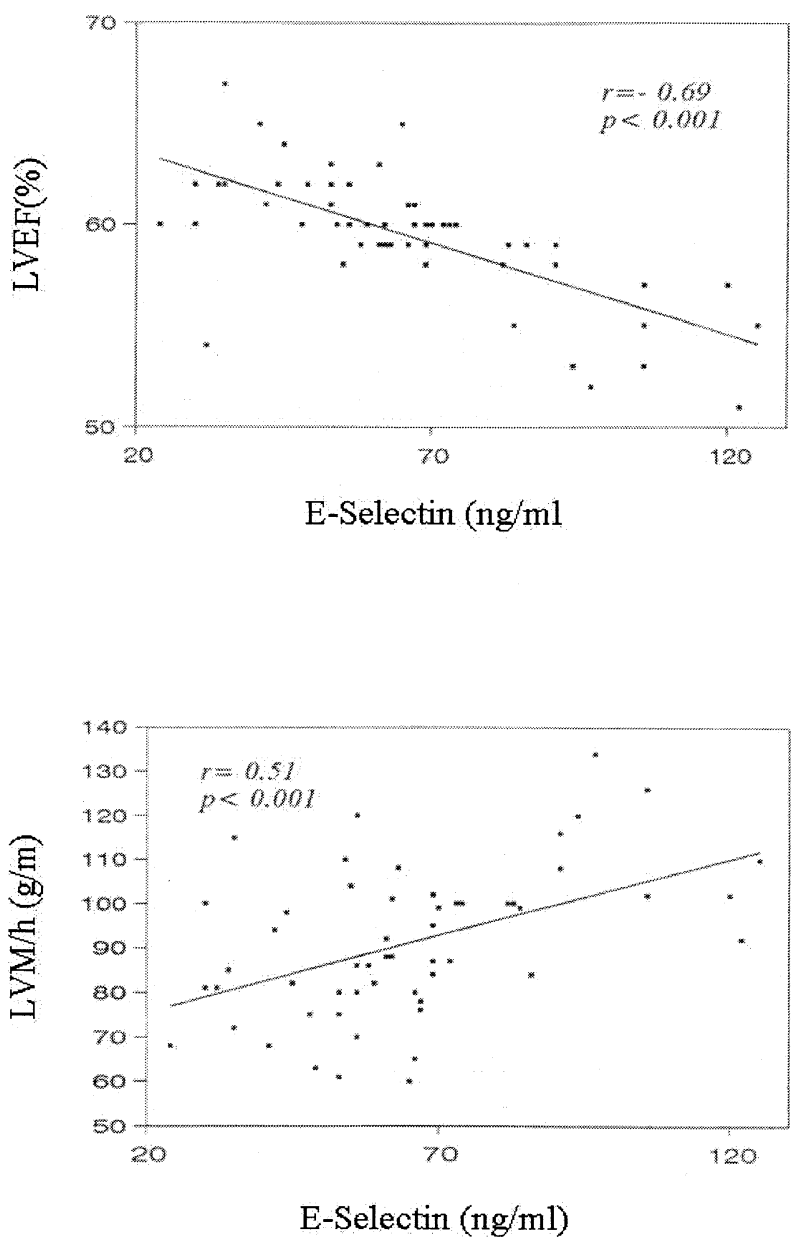

Fig 2. Relationship between sE-S levels, LVEF, andLVM/h in all subjects studied.

Communities Study (ARIC) ${ }^{16}$ indicate a strong association between concentration of soluble adhesion molecules and coronary heart disease. In particular, the risk of coronary heart disease is 5 times greater in subjects with raised values of soluble ICAM-1, and 2 times greater in those with raised sE-S. More recently, Blankenberg et $\mathrm{al}^{15}$ have reported that levels of soluble vascular cell adhesion molecule-1 (VCAM-1), ICAM-1, and E-selectin were significantly related to future death from cardiovascular causes in patients with documented coronary artery disease. These authors suggested that measure- ments of soluble adhesion molecules might provide a better diagnostic tools for new therapeutic strategies.

In our opinion, these contrasting data might be attributable to the different design and goal of the studies. To the best our knowledge, the present study is the first to consider the association between central obesity, E-selectin, ACE genotype, and early carotid and left ventricular abnormalities.

This might represent an interesting issue, since several common human vascular diseases are in part a consequence of the same responses of the endothelium to stress. The prolonged or exaggerated endothelial activation leads to dysfunction that is an early, often preclinical component of vascular disease. ${ }^{10}$

Since we wanted to focus on patients with central obesity by evaluation of the role of body fat distribution and other cardiovascular risk factors, we selected a population of subjects aged less than 40 years and without major cardiovascular risk factors to avoid inclusion of subjects with detectable vascular damage.

Our results that higher BMI and WHR values may be detectable in the group with higher sE-S are in agreement with recent data indicating that obesity per se promotes early endothelial activation and that weight loss counteracts such endothelial activation. ${ }^{17}$ In addition, it has to be emphasized that even if the differences in BMI and WHR values between the 2 groups were small, they have a relevant not only statistical but also biological significance. This is in agreement with our previous $^{4,5,7,8,25}$ and with recent epidemiological results, ${ }^{37}$ indicating that also small changes in BMI and WHR values may assume an important prognostic significance related to cardiovascular risk stratification. In particular, these data support the existence of a continous gradient of cardiovascular risk with increasing BMI and WHR values in obese subjects. This finding might be explained by the known relationship between insulin resistance and central obesity. ${ }^{4}$ In fact, the prevalence of subjects with insulin resistance was higher in the group with higher sE-S and with higher BMI and WHR values than in the group with lower sE-S. Moreover, the recognition of an higher prevalence of subjects with insulin resistance and DD genotype might also explain the higher values of TPR, MBPc, and 24-hour MBP in central obese subjects with higher levels of sE-S. The concomitant relationship between sE-S, BMI, WHR, and blood pressure indicates a strong association between endothelial dysfunction, degree of obesity, central fat distribution, and risk of hypertension.

The abnormalities observed in carotid morphology and in LVM and function in young central obese subjects with higher values of sE-S suggest that these subjects may be characterized by early manifestations of cardiac dysfunction and carotid

Table 3. Correlation Coefficient in Multiple Regression Analysis: Data From Patients With DD Genotype

\begin{tabular}{ccccccc}
\hline & \multicolumn{3}{c}{ Partial Correlation Coefficient } & Multiple Correlation \\
\cline { 2 - 5 } & LVM/h & LVEF & Age & BMI & MBP/24h \\
\hline E-selectin $(\mathrm{ng} / \mathrm{mL})$ & $0.602^{*}$ & $-0.710^{*}$ & 0.122 & 0.210 & 0.221 & $0.589 *$ \\
\hline
\end{tabular}

NOTE. Explanatory variables were left ventricular mass/height (LVM/h), left ventricular ejection fraction (LVEF), age, body mass index (BMI) and 24-hour mean blood pressure (MBP/24h). The first value for partial coefficient, for example, is the correlation coefficient between E-selectin and $\mathrm{LVM} / \mathrm{h}$ after adjustement for age, BMI, and MBP/24h.

${ }^{*} P<.05$. 
atherosclerosis. In fact, it is known that increased LVM and carotid IMWT are independent predictors both of cardiac and cerebrovascular events. ${ }^{37-39}$ The reasons of this association are still unknown but insulin resistance and deletion in ACE gene polymorphism might explain it. Accordingly, in our study population the higher prevalence of subjects with insulin resistance and DD genotype was associated with higher sE-S levels. In addition, 2h-IRI correlated directly with sE-S, indicating that insulin resistance might assume a relevant role in the development of endothelial dysfunction in central obese subjects. This finding is in agreement with recent data of Steinberg et $\mathrm{al}^{40}$ indicating that obesity, rather than hyperglycemia, has a more potent detrimental effect on endothelium-dependent vasodilation. It has been related to a defect in the physiological action of insulin to enhance endothelium-dependent NO synthesis release.

Therefore, to ascribe a defect in endothelial function to obesity/insulin resistance, it is important to rule out other causal associated factors. Accordingly, in this study we excluded central obese subjects with diabetes, hypercholesterolemia, and hypertension. The mechanism by which insulin influences endothelial function is not completely known but it might change endothelium-dependent vasodilation-vasoconstriction balance. ${ }^{41}$ On the other hand, the strong association between the D allele and higher sE-S extends previous data indicating an impaired endothelium-dependent vasodilation in healthy young subjects with the DD genotype. ${ }^{22}$ Unfortunately, the current study was not designed to explain the mechanism responsible of the association between D allele and endothelial dysfunction. This finding may be interesting and it might suggest that genetic factors contribute to characterize cardiovascular risk profile of central obese subjects. Nevertheless, the sample of subjects with II and DI genotype was small to permit a strong conclusion; the importance of this association in the evaluation of cardiac damage in central obese subjects was supported by multiple regression analysis indicating that $\mathrm{sE}-\mathrm{S}$ remains independently related to $L V M / h$ and LVEF only in subjects with the DD genotype. On the contrary, our data seem to exclude that circulating E-selectin levels and D allele are both unrelated expressions of endothelial injury in this population.

In conclusion, our data contribute to stress the role of $\mathrm{sE}-\mathrm{S}$ in central obesity. Accordingly, central obese subjects with higher levels of sE-S and homozygous for the D allele of ACE may be characterized by a higher susceptibility to cardiovascular disease or events in the future. The interest of these endothelial and genetic markers will be strictly dependent on the possibility of a better diagnostic and prognostic stratification. For these reasons, additional prospective data are needed to confirm this assumption, but a prolonged clinical and laboratory follow-up study may be recommended in this particular subset of central obese subjects.

\section{REFERENCES}

1. Manson JF, Colditz GA, Stampfer KI, et al: A prospective study of obesity and risk of coronary heart disease in women. N Engl J Med 29:882-889, 1990

2. Calle EE, Thun MJ, Petrelli JM, et al: Body-mass index and mortality in a prospective cohort of US adults. N Engl J Med 341: 1097-1105, 1999

3. Eckel RH: Obesity in heart disease. A statement for health care professionals from The Nutrition Committee, American Heart Association. Circulation 96:3248-3250, 1997

4. Licata G, Corrao S, Parrinello G, et al: Obesity and Cardiovascular Diseases. Excerpta Medica 1996, pp 5-166

5. Licata G, Scaglione R, Avellone G, et al: Haemostatic function in young subjects with central obesity: Relationship with left ventricular function. Metabolism 44:1417-1421, 1995

6. Licata G, Volpe M, Scaglione R, et al: Salt regulating hormones in young normotensive obese subjects: Effects of saline load. Hypertension 23(Suppl 1):20-22, 1994

7. Licata G, Scaglione R, Barbagallo M, et al: Effect of obesity on left ventricular function studied by radionuclide angiocardiography. Int J Obes 15:295-302, 1991

8. Scaglione R, Dichiara MA, Indovina A, et al: Left ventricular diastolic and systolic function in normotensive obese subjects: Influence of degree and duration of obesity. Eur Heart J 13:738-742, 1992

9. Cines DB, Pollak ES, Buck CA: Endothelial cells in physiology and in pathophysiology of vascular disorders. Blood 91:3527-3561, 1998

10. Yasue H, Matsuyama K, Matsuyama K, et al: Response to angiographically normal human coronary arteries to intracoronary injection of acetylcholine by age and segment: Possible role of early coronary atherosclerosis. Circulation 81:482-490, 1990

11. Schmieder JS: Impaired endothelial function in arterial hypertension and hypercolesterolemia: Potential mechanisms and differences. J Hypertens 18:363-374, 2000

12. Johnstone MT, Creager SJ, Scales KM, et al: Impaired endo- thelium dependent vasodilation in patients with insulin-dependent diabetes mellitus. Circulation 88:2510-2514, 1993

13. Pearson JD: Endothelial cell function and thrombosis. Res Clin Haematol 12:329-341, 1999

14. Dong ZM, Chapman SM, Brown AA, et al: The combined role of P- and E-selectins in atherosclerosis. J Clin Invest 102:145-152, 1998

15. Blankenberg S, Rupprecht HJ, Bickel C, et al: Circulating cell adhesion molecules and death in patients with coronary artery disease. Circulation 104:1336-1342, 2001

16. Hwang S: Circulating adhesion molecules VCAM-1, ICAM-1, and E-selectin in carotid atherosclerosis and incident coronary heart disease case: The Atherosclerosis Risk in Communities (ARIC) Study. Circulation 96:4219-4225, 1997

17. Ferri C, Desideri G, Valenti M, et al: Early upregulation of endothelial adhesion molecules in obese hypertensive men. Hypertension 34:568-573, 1999

18. Cambien F, Poirier O, Lecerf L, et al: Deletion polymorphism in the gene for angiotensin-converting enzyme is a potent risk factor for myocardial infarction. Nature 359:641-644, 1992

19. Lindpaintner K, Pfeffer M.A, Kreutz R, et al: A prospective evaluation of an angiotensin-converting enzyme gene polymor-phism and the risk of ischaemic heart disease. N Engl J Med 332:706-711, 1995

20. Ihnken R, Verho K, Gross M, et al: Deletion polymorphism of the angiotensin-converting enzyme gene is associated with increased plasma angiotensin-converting enzyme activity but not with increased risk for myocardial infarction and coronary artery disease. Ann Intern Med 125:19-25, 1996

21. Di Chiara T, Duro G, Parrinello G, et al: Angiotensin converting enzyme gene polymorphism and central obesity: relationship with blood pressure and left ventricular structure and function. Am J Hypertens 14:A163, 2001 (suppl 1, abstr)

22. Butler R, Morris AD, Burchell B, et al: DD angiotensin-con- 
verting enzyme gene polymorphism is associated with endothelial dysfunction in normal humans. Hypertension 33:11164-1168, 1999

23. Crepaldi G, Belfiore S, Bosello O, et al: Special report: Italian Consensus Conference-Overweight, Obesity and Health. Int J Obes 15:781-790, 1991

24. Licata G, Scaglione R, Ganguzza A, et al: Central obesity and hypertension. Relationship between fasting serum insulin, plasma renin activity and diastolic blood pressure in young obese subjects. Am J Hypertens 7:314-320, 1994

25. Licata G, Scaglione R, Corrao S, et al: Heredity and obesity associated hypertension: Impact of hormonal characteristics and left ventricular mass. J Hypertens 13:611-618, 1995

26. Triolo G, Accardo Palumbo A, Triolo G, et al: Enhancement of endothelial cell E-selectin expression by sera from patients with active Behcet's disease: Moderate correlation with anti-endothelial cell antibodies and serum myeloperoxidase levels. Clin Immunol 91:330-337, 1999

27. John S, Jacobi J, Delles C, et al: Plasma soluble adhesion molecules and endothelium-dependent vasodilation in early human atherosclerosis. Clin Sci 98:521-529, 2000

28. O'Hanlon DM, Fitzsimons H, Lynch J, et al: Soluble adhesion molecules (E-selectin, ICAM-1 and VCAM-1) in brest carcinoma. Eur J Cancer 38:2252-2257, 2002

29. Licata G, Scaglione R, Capuana G, et al: Hypertension in obese subjects: Distinct hypertensive subgroup. J Hum Hypertens 4:37-41, 1990

30. Scaglione R, Corrao S, Ganguzza A, et al: Central obesity, sodium balance and hypertension: Effects of insulin resistance on salt regulating hormone responses to saline load. Endocrinol Metab 4:409416, 1997

31. Corrao S, Paterna S, Arnone S, et al: Two-dimensional echocardiographic evaluation of left ventricular ejection fraction by the ellipsoid single plane algorithm: A reliable method for assessing low of very low ejection fraction values? Cardiology 86:503-507, 1995

32. Barbagallo CM, Pinto A, Gallo S, et al: Carotid atherosclerosis in renal transplant recipients. Transplantation 67:366-371, 1999

33. Nageh MF, Sandberg ET, Marotti KR, et al: Deficiency of inflammatory cell adhesion molecules protects against atherosclerosis in mice. Arterioscler Thromb Vasc Biol 17:1517-1520, 1997

34. Collins RG, Velji R, Guevara NV, et al: P-selectin or intercellular adhesion molecule (ICAM)-1 deficiency substanzially protects against atherosclerosis in apolipoprotein E-deficient mice. J Exp Med 191:189-194, 2000

35. Gearing AJ, Newman W: Circulating adhesion molecules in disease. Immunol Today 14:506-512, 1993

36. Malik I, Danesh J, Whincup P, et al: Soluble adhesion molecules and prediction of coronary heart disease: A prospective study and meta-analysis. Lancet 358:971-976, 2001

37. Kenchaiah S, Evans JC, Levy D, et al: Obesity and the risk of heart failure. N Engl J Med 347:305-313, 2002

38. Muiesan ML, Salvetti M, Rizzoni D, et al: Persistence of left ventricular hypertrophy is a strong indicator of cardiovascular events than baseline left ventricular mass or systolic performance: 10 years of follow-up. J Hypertens 14:443-449, 1996 (suppl 5)

39. Rothwel PM: Carotid artery disease and the risk of ischemic stroke and coronary vascular events. Cerebrovasc Dis 10:21-33, 2000 (suppl 5)

40. Steinberg HO, Chaker H, Leaming R, et al: Obesity/insulin resistance is associated with endothelial dysfunction. J Clin Invest 97:2601-2610, 1996

41. Steinberg HO, Brechtel G, Johnson A, et al: Insulin-mediated skeletal muscle vasodilation is nitric oxide dependent. J Clin Invest 94:1172-1179, 1994 